\title{
ON POLYNOMIAL EIGENFUNCTIONS FOR A CLASS OF DIFFERENTIAL OPERATORS
}

\author{
TANJA Bergkvist AND HANs Rullgi̊nd
}

\section{Introduction}

Jacobi polynomials are solutions of the differential equation

$$
\left(z^{2}-1\right) f^{\prime \prime}(z)+(a z+b) f^{\prime}(z)+c f(z)=0,
$$

where $a, b, c$ are constants satisfying $a>b, a+b>0$ and $c=n(1-a-n)$ for some nonnegative integer $n$. It is a classical fact that the zeros of the Jacobi polynomials lie in the interval $[-1,1]$, and that their density in this interval is proportional to $1 / \sqrt{1-|z|^{2}}$ in the limit when the degree $n$ tends to infinity. The usual proof of this statement involves the observation that, for fixed $a$ and $b$, the Jacobi polynomials constitute an orthogonal system of polynomials with respect to a certain weight function on the interval $[-1,1]$. The desired conclusion then follows from the general theory of orthogonal systems of polynomials.

The following appears to be a natural generalization of the differential equation (1). Let $k \geq 2$ be an integer, and let $Q_{0}, \ldots, Q_{k}$ be polynomials in one complex variable satisfying $\operatorname{deg} Q_{j} \leq j$ with equality when $j=k$. Moreover, we make a normalization by assuming that $Q_{k}$ is monic. Consider the differential operator

$$
T_{Q}(f)=\sum_{j=0}^{k} Q_{j} f^{(j)}
$$

where $f^{(j)}$ denotes the $j$ th derivative of $f$. Operators of this type appear for example in the theory of Bochner-Krall systems of orthogonal polynomials, see [3]. This operator was studied by G. Masson and B. Shapiro in [4]. Particular attention was given the more special operators $T^{\prime}(f)=Q_{k} f^{(k)}$ and $T^{\prime \prime}(f)=$ $(d / d z)^{k}\left(f(z) Q_{k}(z)\right)$. These are indeed special cases of (2) obtained by taking $Q_{j}=0$ or $Q_{j}=\left(\begin{array}{c}k \\ j\end{array}\right) Q_{k}^{(k-j)}$ respectively, for $j=0, \ldots, k-1$. The following result, which shows that $T_{Q}$ has plenty of polynomial eigenfunctions, was proved for the operators $T^{\prime}$ and $T^{\prime \prime}$ in [4].

Theorem 1. For all sufficiently large integers $n$ there is a unique constant $\lambda_{n}$ and a monic polynomial $p_{n}$ of degree $n$ which satisfy

$$
T_{Q}\left(p_{n}\right)=\lambda_{n} p_{n} \text {. }
$$

Moreover, we have $\lambda_{n} / n(n-1) \ldots(n-k+1) \rightarrow 1$ when $n \rightarrow \infty$.

Received October 22, 2001. 
G. Masson and B. Shapiro made a number of striking conjectures, based on numerical evidence, about the zeros of the eigenpolynomials $p_{n}$. They also observed that when $k>2$, the sequence $p_{n}$ is in general not an orthogonal system of polynomials, so they cannot be studied by means of the extensive theory known for such systems.

The goal of this note is to prove some of the conjectures in [4]. More precisely, we shall show that in the limit when $n \rightarrow \infty$, the zeros of $p_{n}$ are distributed according to a certain probability measure. This probability measure depends only on the "leading polynomial" $Q_{k}$ and may be independently characterized in the following way.

Theorem 2. Let $Q_{k}$ be a monic polynomial of degree $k$. Then there exists a unique probability measure $\mu_{Q_{k}}$ with compact support whose Cauchy transform

$$
C(z)=\int \frac{d \mu_{Q_{k}}(\zeta)}{z-\zeta}
$$

satisfies $C(z)^{k}=1 / Q_{k}(z)$ for almost all $z \in \mathbf{C}$.

We record some properties of the measure $\mu_{Q_{k}}$ which will be encountered in the proof of Theorem 2. Let $\operatorname{supp} \mu$ denote the support of a measure $\mu$. Also, let

$$
\Psi(z)=\int Q_{k}(z)^{-1 / k} d z
$$

be a primitive function of $Q_{k}(z)^{-1 / k}$. At this point, we think of $\Psi$ as a locally defined function in any simply connected domain where $Q_{k}$ does not vanish. The choice of a branch of $Q_{k}(z)^{1 / k}$ and an integration constant is of no importance here. As need arises, specifications will be made concerning these choices.

Theorem 3. Let $Q_{k}$ and $\mu_{Q_{k}}$ be as in Theorem 2. Then supp $\mu_{Q_{k}}$ is the union of finitely many smooth curve segments, and each of these curves is mapped to a straight line by the mapping $\Psi$. Moreover, supp $\mu_{Q_{k}}$ contains all the zeros of $Q_{k}$, is contained in the convex hull of the zeros of $Q_{k}$ and is connected and has connected complement.

If $p$ is a polynomial of degree $n$, we can construct a probability measure $\mu$ by placing a point mass of size $1 / n$ at each zero of $p$. We will call $\mu$ the root measure of $p$. Our main result is

Theorem 4. Let $p_{n}$ be the monic degree $n$ eigenpolynomial of the operator $T_{Q}$ and let $\mu_{n}$ be the root measure of $p_{n}$. Then $\mu_{n}$ converges weakly to $\mu_{Q_{k}}$ when $n \rightarrow \infty$.

To illustrate, we show the zeros of the eigenpolynomial $p_{40}$ for the degree 5 operator $T_{Q}$ with $Q_{5}(z)=z(z-1+i)(z-5)(z-2-4 i)(z-4-4 i)$ and $Q_{0}=\cdots=Q_{4}=0$. Large dots represent the zeros of $Q_{5}$ (which are, in this case, also zeros of $p_{n}$ ) and small dots represent (the remaining) zeros of $p_{40}$. It is remarkable how well the zeros of the eigenpolynomial line up along the curves predicted by our results. Notice also how these curves are straightened out by the mapping $\Psi$. 

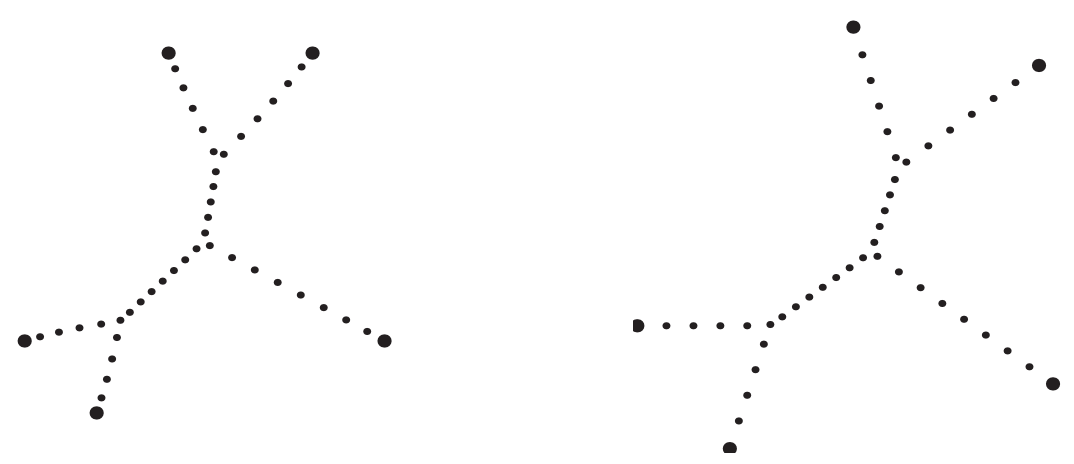

FiguRE 1. Zeros of the polynomial $Q_{5}$ and the eigenpolynomial $p_{40}$ (left) and the image of these zeros under a branch of the mapping $\Psi$.

It is not difficult to deduce various other features of the measure $\mu_{Q_{k}}$ from the properties given in Theorem 3 and the defining property (4). For example, a recipe for computing the angles between the different curve segments is conjectured in [4]. The correctness of the procedure follows easily from our results. We refrain from going into details, but the key observation is the following. Suppose $z_{0}$ is a point on one of the curve segments in $\operatorname{supp} \mu_{Q_{k}}$ and let $C_{1}$ and $C_{2}$ be the limiting values of $C(z)$ as $z$ approaches $z_{0}$ from different sides of the curve. Then $C_{1}$ and $C_{2}$ are $k$ th roots of $1 / Q_{k}\left(z_{0}\right)$, and their actual values are easily found if the combinatorics of $\operatorname{supp} \mu_{Q_{k}}$ are known (which was assumed in the recipe mentioned above). From the fact that $\pi \mu_{Q_{k}}=\partial C / \partial \bar{z} \geq 0$, it follows that the curve must be perpendicular to $\bar{C}_{1}-\bar{C}_{2}$ at $z_{0}$. Using this observation where several curves meet, it is possible to deduce the angles between them. Notice also that the density of $\mu_{Q_{k}}$ at $z_{0}$ is proportional to $\left|C_{1}-C_{2}\right|$.

It is particularly easy to compute $\mu_{Q_{k}}$ when $Q_{k}$ has only real zeros. Denote the zeros by $z_{1}, \ldots, z_{k}$ in increasing order. From Theorem 3 we know that $\operatorname{supp} \mu_{Q_{k}}=\left[z_{1}, z_{k}\right]$. A direct computation shows that on the subinterval $\left[z_{j}, z_{j+1}\right]$, the measure is given by

$$
\mu_{Q_{k}}=\frac{1}{\pi} \frac{\partial C}{\partial \bar{z}}=\frac{2}{\pi\left|Q_{k}\right|^{1 / k}} \sin \left(\frac{\pi j}{k}\right) d x
$$

where $d x$ denotes Lebesgue measure on the real line. This remains true even if $Q_{k}$ has multiple zeros, except if all the zeros coincide. In this case, $\mu_{Q_{k}}$ reduces, of course, to a point mass at this multiple zero.

Let us finally discuss some possible applications and directions for further research. As we already mentioned, operators of the type we consider occur in the theory of Bockner-Krall orthogonal systems. More precisely, a Bochner-Krall system (BKS), is a sequence of polynomials $p_{n}$ which are both eigenpolynomials of an operator $T_{Q}$ (here one omits the assumption that $\operatorname{deg} Q_{k}=k$ ) and also orthogonal with respect to a suitable inner product. It is a long standing problem to classify all BKS. A great deal is known about the asymptotic distribution of 
zeros of orthogonal polynomials. By comparing such results with our results on the distribution of zeros of eigenpolynomials, we believe that it will be possible to gain new insight into the nature of BKS. To get the most out of this approach, however, it would be desirable to have generalizations of our results to the case $\operatorname{deg} Q_{k}<k$. Computer experiments performed by the first author indicate that a limiting measure exists in this case too, but that it may not have compact support.

This paper is organized as follows. In section 2 we compute the matrix for the operator $T_{Q}$ with respect to the basis of monomials $1, z, z^{2}, \ldots$, and use this to prove Theorem 1. Section 3 contains a proof of the uniqueness part of Theorem 2. Along the way, we also prove essentially all the statements in Theorem 3 . In section 4 we recall some basic facts on the weak topology of measures in the complex plane and on logarithmic potentials and Cauchy transforms. We also outline the connection of these concepts to root measures of polynomials and prove a general result on the relation between the zeros of a polynomial and those of its derivative. In the final section 5 we apply the ideas from the previous section to give a proof of Theorem 4 . The existence part of Theorem 2 is also a consequence of this proof.

Acknowledgements. The authors are sincerely grateful to Harold Shapiro for highly valuable comments and discussions, and in particular for suggesting the use of the Cauchy transform. We would also like to thank Gisli Masson and Boris Shapiro for introducing us to the problem and for their support during our work.

\section{Calculation of the matrix}

Recall that the differential operator $T_{Q}$ is defined by

$$
T_{Q}=Q_{k} \frac{d^{k}}{d z^{k}}+Q_{k-1} \frac{d^{k-1}}{d z^{k-1}}+\cdots+Q_{1} \frac{d}{d z}+Q_{0}
$$

where the $Q_{m}$ are polynomials such that $\operatorname{deg} Q_{m} \leq m$ for $m=0, \ldots, k$ and $\operatorname{deg} Q_{k}=k$. Let $p_{n}(z)=\sum_{i=0}^{n} a_{n, i} z^{i}$ be a monic polynomial of degree $n$ and let $Q_{m}(z)=\sum_{j=0}^{m} q_{m, j} z^{j}$. Using these notations we get

$$
\begin{aligned}
T_{Q}\left(p_{n}\right) & =\sum_{m=0}^{k} Q_{m} \cdot \frac{d^{m}}{d z^{m}} p_{n}=\sum_{m=0}^{k}\left[\sum_{j=0}^{m} q_{m, j} z^{j}\right] \cdot\left[\sum_{i \geq m}^{n} a_{n, i} \cdot \frac{i !}{(i-m) !} z^{i-m}\right]= \\
& =\sum_{m=0}^{k} \sum_{s=0}^{n}\left[\sum_{\substack{s=j+i-m \\
m \leq i \leq n \\
0 \leq j \leq m}} q_{m, j} \cdot a_{n, i} \cdot \frac{i !}{(i-m) !}\right] z^{s}= \\
& =\sum_{s=0}^{n}\left[\sum_{\substack { k=0 \\
\begin{subarray}{c}{k=j+i-m \\
m \leq i \leq n \\
0 \leq j \leq m{ k = 0 \\
\begin{subarray} { c } { k = j + i - m \\
m \leq i \leq n \\
0 \leq j \leq m } }\end{subarray}} q_{m, j} \cdot a_{n, i} \cdot \frac{i !}{(i-m) !}\right] z^{s} .
\end{aligned}
$$


Lemma 1. If $p_{n}$ is monic and $T_{Q}\left(p_{n}\right)=\lambda_{n} \cdot p_{n}$ then

$$
\lambda_{n}=\sum_{m=0}^{k} q_{m, m} \cdot \frac{n !}{(n-m) !} .
$$

Proof. With $p_{n}$ monic and $T_{Q}\left(p_{n}\right)=\lambda_{n} \cdot p_{n}=\lambda_{n} z^{n}+\lambda_{n} \cdot a_{n, n-1} z^{n-1}+\ldots+$ $\lambda_{n} \cdot a_{n, 0}$, finding the eigenvalue $\lambda_{n}$ amounts to finding the coefficient at $z^{n}$ in $T_{Q}\left(p_{n}\right)$. Note that $\operatorname{deg} Q_{m} \frac{d^{m}}{d z^{m}} p_{n} \leq m+n-m=n$ with equality if and only if $\operatorname{deg} Q_{m}=m$. Thus we can assume that $p_{n}=z^{n}$ (since any lower degree terms of $p_{n}$ will result in terms of degree lower than $n$ in $\left.T_{Q}\left(p_{n}\right)\right)$. We therefore consider

$$
\begin{aligned}
T_{Q}\left(z^{n}\right) & =\sum_{m=0}^{k} Q_{m} \cdot \frac{d^{m}}{d z^{m}} z^{n}=\sum_{m=0}^{k} Q_{m} \cdot \frac{n !}{(n-m) !} z^{n-m}= \\
& =\sum_{m=0}^{k}\left[\left(\sum_{j=0}^{m} q_{m, j} z^{j}\right) \cdot \frac{n !}{(n-m) !} z^{n-m}\right]= \\
& =\sum_{m=0}^{k}\left[\sum_{j=0}^{m} q_{m, j} \cdot \frac{n !}{(n-m) !} z^{j+n-m}\right] .
\end{aligned}
$$

Setting $j=m$ we get

$$
\lambda_{n} z^{n}=\sum_{m=0}^{k} q_{m, m} \cdot \frac{n !}{(n-m) !} z^{n} \quad \Longrightarrow \quad \lambda_{n}=\sum_{m=0}^{k} q_{m, m} \cdot \frac{n !}{(n-m) !} .
$$

Lemma 2. For $n \geq 1$ the coefficient vector $X$ of $p_{n}$ with components $a_{n, 0}, \ldots, a_{n, n-1}$ satisfies the linear system $M X=Y$, where $Y$ is a vector and $M$ is an upper triangular matrix, both with entries expressible in the coefficients $q_{m, j}$ (see below).

Proof. The relation

$$
T_{Q}\left(p_{n}\right)=\lambda_{n} \cdot p_{n}
$$

is equivalent to

$$
\sum_{s=0}^{n}\left[\sum_{\substack { m=0 \\
\begin{subarray}{c}{s=j+i-m \\
m \leq i \leq n \\
0 \leq j \leq m{ m = 0 \\
\begin{subarray} { c } { s = j + i - m \\
m \leq i \leq n \\
0 \leq j \leq m } }\end{subarray}}^{k} q_{m, j} \cdot a_{n, i} \cdot \frac{i !}{(i-m) !}\right] z^{s}=\lambda_{n} \sum_{s=0}^{n} a_{n, s} z^{s}
$$

With $j=m+s-i$ the condition $j \leq m$ gives $i \geq s$ and the condition $j \geq 0$ results in $m \geq i-s$. Therefore the above system will be equivalent to

$$
\sum_{s=0}^{n}\left[\sum_{s \leq i \leq n} \sum_{i-s \leq m \leq \min (i, k)} q_{m, m+s-i} \cdot \frac{i !}{(i-m) !} \cdot a_{n, i}-\lambda_{n} \cdot a_{n, s}\right] z^{s}=0 .
$$


Thus for each $s$ we have

$$
\sum_{s \leq i \leq n} \sum_{i-s \leq m \leq \min (i, k)} q_{m, m+s-i} \cdot \frac{i !}{(i-m) !} \cdot a_{n, i}-\lambda_{n} \cdot a_{n, s}=0
$$

or, equivalently,

$$
\begin{gathered}
\sum_{s \leq i \leq n-1} \quad \sum_{i-s \leq m \leq \min (i, k)} q_{m, m+s-i} \cdot \frac{i !}{(i-m) !} \cdot a_{n, i}-\lambda_{n} \cdot a_{n, s}= \\
=\sum_{n-s \leq m \leq \min (n, k)} q_{m, m+s-n} \cdot \frac{n !}{(n-m) !} \cdot a_{n, n}
\end{gathered}
$$

where $a_{n, n}=1$. The $n \times n$ matrix $M$ is thus constructed for $0 \leq s \leq n-1$ and $0 \leq i \leq n-1$. The left-hand side of the above equation corresponds to the $(s+1)$ st row in $M$ multiplied by the column vector $X$, and the right-hand side represents the $(s+1)$ st row in $Y$. Thus the entries of $M$ are given by

$$
M_{s+1, i+1}=\sum_{i-s \leq m \leq \min (i, k)} q_{m, m+s-i} \cdot \frac{i !}{(i-m) !}-\lambda_{n} \cdot \delta_{i, s}
$$

where $\delta$ denotes the Kronecker delta. The condition $i \geq s$ implies that $M$ is upper triangular.

We can now prove Theorem 1 . Using Lemma 1 we get

$$
\begin{aligned}
& \frac{\lambda_{n}}{n(n-1) \ldots(n-k+1)}=\frac{\sum_{m=0}^{k} q_{m, m} \cdot \frac{n !}{(n-m) !}}{n(n-1) \ldots(n-k+1)}= \\
& =\frac{q_{0,0} \frac{n !}{n !}+q_{1,1} \frac{n !}{(n-1) !}+q_{2,2} \frac{n !}{(n-2) !}+\ldots+q_{k-1, k-1} \frac{n !}{(n-k+1) !}+q_{k, k} \frac{n !}{(n-k) !}}{n(n-1) \ldots(n-k+1)}= \\
= & \frac{q_{0,0}}{n(n-1) \ldots(n-k+1)}+\frac{q_{1,1}}{(n-1) \ldots(n-k+1)}+\ldots+\frac{q_{k-1, k-1}}{(n-k+1)}+q_{k, k} .
\end{aligned}
$$

Thus

$$
\lim _{n \rightarrow \infty} \frac{\lambda_{n}}{n(n-1) \ldots(n-k+1)}=q_{k, k}=1,
$$

and the first part of Theorem 1 is proved. To prove the uniqueness part, we show that the determinant of the matrix $M$ constructed in Lemma 2 is non-zero for sufficiently large values of $n$. Since the matrix is upper triangular its determinant equals the product of the diagonal elements. Thus it suffices to prove that for sufficiently large $n$ every diagonal element is non-zero.

The diagonal element $M_{i+1, i+1}$ of $M$ is obtained by letting $i=s$ in (5) and so

$$
M_{i+1, i+1}=\sum_{0 \leq m \leq \min (i, k)} q_{m, m} \cdot \frac{i !}{(i-m) !}-\lambda_{n}
$$


for $i=0, \ldots, n-1$. But the last expression equals $\lambda_{i}-\lambda_{n}$. Indeed, if $i \geq k$ then

$$
\sum_{0 \leq m \leq \min (i, k)} q_{m, m} \cdot \frac{i !}{(i-m) !}=\sum_{0 \leq m \leq k} q_{m, m} \cdot \frac{i !}{(i-m) !}=\lambda_{i} .
$$

If $i<k$ then this is again true since by definition $i ! /(i-m) !=0$ for $i<m \leq k$. Thus we have to show that $\lambda_{i}-\lambda_{n} \neq 0 \quad \forall i<n$ as $n \rightarrow \infty$. For small values of $i$ (for example, $i<k$ ) we have $\lambda_{i}<\infty$ and $\lambda_{n} \rightarrow \infty$ as $n \rightarrow \infty$, implying $\lambda_{i}-\lambda_{n} \neq 0$. For larger values of $i(0<m<k \leq i)$ we get

$$
\begin{aligned}
\lambda_{n}-\lambda_{i} & =\sum_{m=0}^{k} q_{m, m} \frac{n !}{(n-m) !}-\sum_{m=0}^{k} q_{m, m} \frac{i !}{(i-m) !}= \\
& =\sum_{m=0}^{k} q_{m, m}\left[\frac{n !}{(n-m) !}-\frac{i !}{(i-m) !}\right] .
\end{aligned}
$$

Dividing the last expression by $\frac{n !}{(n-k) !}-\frac{i !}{(i-k) !}$ we obtain

$$
\frac{\lambda_{n}-\lambda_{i}}{\frac{n !}{(n-k) !}-\frac{i !}{(i-k) !}}=q_{k, k}+\sum_{m=1}^{k-1} q_{m, m} \frac{\frac{n !}{(n-m) !}-\frac{i !}{(i-m) !}}{\frac{n !}{(n-k) !}-\frac{i !}{(i-k) !}} .
$$

which tends to $q_{k, k}=1$ as $n \rightarrow \infty$, since for each $m \leq k-1$ we get

$$
\begin{aligned}
\lim _{n \rightarrow \infty} \frac{\frac{n !}{(n-m) !}-\frac{i !}{(i-m) !}}{\frac{n !}{(n-k) !}-\frac{i !}{(i-k) !}} & =\lim _{n \rightarrow \infty} \frac{\frac{i !}{(n-m) !}\left(\frac{n !}{i !}-\frac{(n-m) !}{(i-m) !}\right)}{\frac{i !}{(n-k) !}\left(\frac{n !}{i !}-\frac{(n-k) !}{(i-k) !}\right)} \\
& =\lim _{n \rightarrow \infty} \frac{(n-k) !}{(n-m) !} \cdot \frac{\left(\frac{n !}{i !}-\frac{(n-m) !}{(i-m) !}\right)}{\left(\frac{n !}{i !}-\frac{(n-k) !}{(i-k) !}\right)} \\
& =0 .
\end{aligned}
$$

Therefore $\lambda_{n}-\lambda_{i} \neq 0$. Thus, as $n \rightarrow \infty$, every diagonal element of $M$ becomes non-zero and so its determinant will be non-zero, implying that $M$ is invertible. Thus the system $M X=Y$ will have a unique solution.

Remark. If $\operatorname{deg} Q_{m}=m$ for at least one $m$ (not necessarily $k$ ) and if the coefficients $q_{m, m}$ of all such $Q_{m}$ have equal sign, then there exists a unique monic eigenpolynomial of degree $n$ for every value of $n$. To show this consider as before the determinant of the matrix $M$ :

$$
\operatorname{det} M=\prod_{i=0}^{n-1}\left[\sum_{0 \leq m \leq \min (i, k)} q_{m, m} \cdot \frac{i !}{(i-m) !}-\lambda_{n}\right] .
$$

For $i \geq k$ the $i$ :th factor of this product equals

$$
\sum_{0 \leq m \leq k} q_{m, m}\left[\frac{i !}{(i-m) !}-\frac{n !}{(n-m) !}\right]
$$


This expression is non-zero since $i<n$, and by assumption all the $q_{m, m}$ have equal sign and $q_{m, m} \neq 0$ for at least one $m$. For $i<k$ the $i$ :th factor of $\operatorname{det} M$ equals

$$
\begin{aligned}
& \sum_{0 \leq m \leq i} q_{m, m}\left[\frac{i !}{(i-m) !}-\frac{n !}{(n-m) !}\right]-\sum_{i+1 \leq m \leq k} q_{m, m} \cdot \frac{n !}{(n-m) !}= \\
= & -\sum_{0 \leq m \leq i} q_{m, m}\left[\frac{n !}{(n-m) !}-\frac{i !}{(i-m) !}\right]-\sum_{i+1 \leq m \leq k} q_{m, m} \cdot \frac{n !}{(n-m) !} .
\end{aligned}
$$

This is also non-zero, since $i<n$, all terms have equal sign and at least one term is non-zero. Thus every factor in the product defining the determinant is non-zero and we get a unique solution of $M X=Y$ for every value of $n$.

\section{Probability measures whose Cauchy transform satisfies an algebraic equation}

In this section we will prove the uniqueness part of Theorem 2 and show that the mesure $\mu_{Q_{k}}$, if it exists, has the properties stated in Theorem 3. The proof relies heavily on the following lemma.

Lemma 3. Let $A \subset \mathbf{C}$ be a finite set, $U \subset \mathbf{C}$ a convex domain and $\chi: U \rightarrow A$ a measurable function such that $\partial \chi / \partial \bar{z} \geq 0$ (in the sense of distributions). Let $a \in A, z_{0} \in U$ and assume that $\chi^{-1}(a) \cap\left\{\left|z-z_{0}\right|<r\right\}$ has positive Lebesgue measure for every $r>0$. Then $\chi(z)=a$ almost everywhere in $U \cap\left(z_{0}+\Gamma_{a}\right)$ where

$$
\Gamma_{a}=\{z \in \mathbf{C} ; \operatorname{Re}(a z) \geq \operatorname{Re}(b z), \forall b \in A\} .
$$

Note that if $\chi^{-1}(a) \cap\left\{\left|z-z_{0}\right|<r\right\}$ has positive Lebesgue measure for every $a \in A$ and all $r>0$, then $\chi$ is determined completely (outside a set of measure $0)$ since the cones $\Gamma_{a}$ cover the whole complex plane.

Proof. Let $\chi_{a}$ denote the characteristic function of the set $\chi^{-1}(a)$. We will show that if $z_{1}, z_{2} \in U$ with $z_{2}-z_{1} \in \Gamma_{a}$, and $\phi$ is a positive test function such that $z_{1}+\operatorname{supp} \phi$ and $z_{2}+\operatorname{supp} \phi$ are both contained in $U$, then

$$
\left(\phi * \chi_{a}\right)\left(z_{1}\right) \leq\left(\phi * \chi_{a}\right)\left(z_{2}\right) .
$$

The desired conclusion follows from this. Indeed, let $\phi_{j}$ be a sequence of positive test functions such that $\operatorname{supp} \phi_{j} \rightarrow 0$ and $\int \phi_{j} d \lambda=1$, where $\lambda$ denotes planar Lebesgue measure. We know then that $\phi_{j} * \chi_{a}$ converges in $L_{l o c}^{1}$ to $\chi_{a}$. Hence, for any $\epsilon, r>0$ we can find for all sufficiently large $j$ a point $z_{1}$ with $\left|z_{1}-z_{0}\right|<r$ such that $\left(\phi_{j} * \chi_{a}\right)\left(z_{1}\right)>1-\epsilon$. It follows from $(7)$ that $\left(\phi_{j} * \chi_{a}\right)\left(z_{2}\right)>1-\epsilon$ and hence

$$
\left|\left(\phi_{j} * \chi\right)\left(z_{2}\right)-a\right|=\left|\int \phi_{j}\left(z_{2}-\zeta\right)(\chi(\zeta)-a) d \lambda(\zeta)\right|<\epsilon \max _{b \in A}|b-a|
$$

for all $z_{2} \in z_{1}+\Gamma_{a}$. Letting $\epsilon$ and $r$ tend to 0 and $j \rightarrow \infty$ it follows that $\chi(z)=\lim _{j \rightarrow \infty}\left(\phi_{j} * \chi\right)(z)=a$ for almost all $z$ in $z_{0}+\Gamma_{a}$. 
We now prove the inequality (7). Without loss of generality we may assume that $z_{2}-z_{1}>0$ and that $a=0$, for the general case can be reduced to this situation by replacing $\chi$ with the function $e^{i \theta}\left(\chi\left(e^{i \theta} z\right)-a\right)$ where $\theta=\arg \left(z_{2}-z_{1}\right)$. The assumption that $z_{2}-z_{1} \in \Gamma_{a}$ then implies that $A$ is contained in the closed left half plane $\{\operatorname{Re} z \leq 0\}$.

For any $\epsilon>0$, let $\tilde{\chi}_{\epsilon}=\log (\chi-\epsilon)$ where we have chosen a branch of the logarithm function which is continuous in the left half plane. Let $\psi$ be a positive test function and note that $\partial(\psi * \chi) / \partial \bar{z} \geq 0$ and $\operatorname{Re} \psi * \chi \leq 0$. It follows that

$$
\operatorname{Re} \frac{\partial}{\partial \bar{z}} \log (\psi * \chi-\epsilon)=\operatorname{Re}\left(\frac{1}{\psi * \chi-\epsilon} \cdot \frac{\partial(\psi * \chi)}{\partial \bar{z}}\right) \leq 0 .
$$

When $\operatorname{supp} \psi \rightarrow 0$ with $\int \psi d \lambda=1$, we have that $\log (\psi * \chi-\epsilon) \rightarrow \tilde{\chi}_{\epsilon}$ in $L_{l o c}^{1}$, and hence as a distribution. By passing to the limit it follows that

$$
\operatorname{Re} \frac{\partial \tilde{\chi}_{\epsilon}}{\partial \bar{z}} \leq 0
$$

If we write $\tilde{\chi}_{\epsilon}=\sigma_{\epsilon}+i \tau_{\epsilon}$, this means that

$$
\frac{\partial \sigma_{\epsilon}}{\partial x} \leq \frac{\partial \tau_{\epsilon}}{\partial y}
$$

Fix a positive test function $\phi$ such that $z_{j}+\operatorname{supp} \phi \subset U$ for $j=1,2$ and consider the function $\left(\phi * \sigma_{\epsilon}\right)\left(z_{1}+\xi\right)$ of the real variable $\xi$. It follows from (8) and the fact that $\tau_{\epsilon}$ is uniformly bounded for all $\epsilon$ that

$$
\begin{aligned}
\frac{\partial}{\partial \xi}\left(\phi * \sigma_{\epsilon}\right)\left(z_{1}+\xi\right) & =\int \frac{\partial \phi}{\partial x}\left(z_{1}+\xi-\zeta\right) \sigma_{\epsilon}(\zeta) d \lambda(\zeta) \\
& \leq \int \frac{\partial \phi}{\partial y}\left(z_{1}+\xi-\zeta\right) \tau_{\epsilon}(\zeta) d \lambda(\zeta) \\
& \leq M
\end{aligned}
$$

where the constant $M$ does not depend on $\epsilon$. In particular,

$$
\left(\phi * \sigma_{\epsilon}\right)\left(z_{2}\right)-\left(\phi * \sigma_{\epsilon}\right)\left(z_{1}\right) \leq M\left|z_{2}-z_{1}\right| .
$$

On the other hand it is clear that

$$
\left(\phi * \sigma_{\epsilon}\right)(z)=\log \epsilon \cdot\left(\phi * \chi_{a}\right)(z)+O(1) .
$$

Now (7) follows from (9) and (10) when $\epsilon \rightarrow 0$.

We deduce two corollaries of Lemma 3.

Corollary 1. Let $U \subset \mathbf{C}$ be a convex domain and $A \subset \mathbf{C}$ a finite set. If $v$ is a subharmonic function defined in $U$ such that $2 \partial v / \partial z \in A$ almost everywhere, then $v$ is convex. 
Recall that a subharmonic function can locally be written as the sum of a harmonic function and a logarithmic potential. It follows that the distribution $\partial v / \partial z$ is represented by a locally integrable function. The condition $2 \partial v / \partial z \in A$ should be interpreted by saying that $2 \partial v / \partial z$ is represented by a measurable function with values in $A$.

Proof. Let $\chi=2 \partial v / \partial z$. Since $v$ is subharmonic, $\partial \chi / \partial \bar{z} \geq 0$. Take any point $z_{0} \in U$ and let $A_{0}$ be the set of all $a \in A$ such that $\chi^{-1}(a)$ has positive measure in every neighbourhood of $z_{0}$. Let $U_{0}$ be a convex neighbourhood of $z_{0}$ such that $\chi(z) \in A_{0}$ almost everywhere in $U_{0}$. By Lemma $3, \chi(z)=a$ almost everywhere in $U_{0} \cap\left(z_{0}+\Gamma_{a}\right)$ where $\Gamma_{a}$ is defined by (6) but with $A_{0}$ in place of $A$. This implies that $v(z)=v\left(z_{0}\right)+\operatorname{Re} a\left(z-z_{0}\right)$ for all $z \in U_{0} \cap\left(z_{0}+\Gamma_{a}\right)$, so that

$$
v(z)=v\left(z_{0}\right)+\max _{a \in A_{0}} \operatorname{Re} a\left(z-z_{0}\right), \quad z \in U_{0} .
$$

We have shown that in a neighbourhood of $z_{0}, v$ is the maximum of certain linear functions, hence it is convex there. Since $z_{0}$ was arbitrary, it follows that $v$ is convex.

Corollary 2. Let $A \subset \mathbf{C}$ be a finite set, $U \subset \mathbf{C}$ a convex domain and let $\chi: U \rightarrow A$ be a measurable function. Then $\partial \chi / \partial \bar{z} \geq 0$ if and only if there exist real numbers $c_{a}$ (possibly equal to $-\infty$ ) such that $\chi(z)=$ a almost everywhere in $G_{a}$ where

$$
G_{a}=\left\{z \in U ; c_{a}+\operatorname{Re}(a z) \geq c_{b}+\operatorname{Re}(b z), \forall b \in A\right\}
$$

Proof. Suppose $c_{a}$ are real numbers such that $\chi(z)=a$ almost everywhere in $G_{a}$. Let $v(z)=\max _{a \in A}\left(c_{a}+\operatorname{Re}(a z)\right)$. Then $v$ is subharmonic and $\chi=2 \partial v / \partial z$, hence

$$
\frac{\partial \chi}{\partial \bar{z}}=2 \frac{\partial^{2} v}{\partial z \partial \bar{z}} \geq 0
$$

Suppose conversely that $\partial \chi / \partial \bar{z} \geq 0$. Since $\partial \chi / \partial \bar{z}$ is real, there exists a real valued function $v$ defined in $U$ with $2 \partial v / \partial z=\chi$. It follows from Corollary 1 that $v$ is convex. Moreover, we see from the proof that

$$
v(z)=\max _{a \in A}\left(c_{a}+\operatorname{Re}(a z)\right)
$$

where

$$
c_{a}=\inf _{z \in U}(v(z)-\operatorname{Re}(a z)) .
$$

If we define $G_{a}$ using these constants $c_{a}$ it follows that $v(z)=c_{a}+\operatorname{Re}(a z)$ for $z \in G_{a}$, hence $\chi(z)=2 \partial v / \partial z=a$ in $G_{a}$. 
Fix a monic polynomial $Q_{k}$ of degree $k$ and suppose that $\mu$ is a compactly supported probability measure whose Cauchy transform $C(z)$ satisfies

$$
C(z)^{k}=1 / Q_{k}(z) .
$$

We will first show that $\mu$ has the properties asserted in Theorem 3, except that $\operatorname{supp} \mu_{Q_{k}}$ is contained in the convex hull of the zeros of $Q_{k}$, which will be proved in section 5 .

Lemma 4. If the Cauchy transform of $\mu$ satisfies (11), then the support of $\mu$ is the union of finitely many smooth curve segments. These curves are mapped to lines by $\Psi$.

Proof. It is sufficient to prove that $\operatorname{supp} \mu$ has these properties in a neighbourhood of any given point $z_{0}$. Assume first that $Q_{k}\left(z_{0}\right) \neq 0$. Choose a branch of $Q_{k}(z)^{-1 / k}$ defined in a simply connected neighbourhood of $z_{0}$ and let $\Psi$ be a primitive function of $Q_{k}(z)^{-1 / k}$. Let $U$ be a convex neighbourhood of $\Psi\left(z_{0}\right)$ so small that $\Psi$ maps a neighbourhood of $z_{0}$ bijectively onto $U$. By (11) we can write $C(z)=\chi(\Psi(z)) Q_{k}(z)^{-1 / k}$ for $z \in \Psi^{-1}(U)$, where $\chi$ has values in the set of $k$ th roots of unity. If we write $w=\Psi(z)$, then

$\pi \mu=\frac{\partial C}{\partial \bar{z}}=\frac{\partial \chi(\Psi(z))}{\partial \bar{z}} \cdot Q_{k}^{-1 / k}=\Psi^{*}\left(\frac{\partial \chi}{\partial \bar{w}}\right) \cdot \frac{\overline{\partial \Psi}}{\partial z} \cdot Q_{k}^{-1 / k}=\Psi^{*}\left(\frac{\partial \chi}{\partial \bar{w}}\right) \cdot\left|Q_{k}\right|^{-2 / k}$ where $\Psi^{*}$ denotes the pullback of distributions in $U$ by $\Psi$. Since $\mu$ is positive, it follows that

$$
\frac{\partial \chi}{\partial \bar{w}} \geq 0
$$

By Corollary 2, $U$ is the union of sets $G_{a}$ whose boundaries are finite unions of line segments, such that $\chi$ is constant in each $G_{a}$. It follows that supp $\mu \cap$ $\Psi^{-1}(U)=\Psi^{-1}(\operatorname{supp} \partial \chi / \partial \bar{z})$ is the union of finitely many curve segments which are mapped to straight lines by $\Psi$.

If $z_{0}$ is a zero of $Q_{k}$, we take a disc $D$ centered at $z_{0}$ wich does not contain any other zeros of $Q_{k}$. If $\gamma$ is any ray emanating at $z_{0}$, we can define single valued branches of $Q(z)^{-1 / k}$ and $\Psi$ in $D \backslash \gamma$. Notice that $\Psi$ is continuous up to $z_{0}$. Let $U$ be any half disc centered at $\Psi\left(z_{0}\right)$ and contained in $\Psi(D \backslash \gamma)$. It follows as in the first part of the proof that $\operatorname{supp} \mu$ has the required properties in $\Psi^{-1}(U)$. By varying $\gamma$ and $U$, we see that the same holds in a full neighbourhood of $z_{0}$.

Hence $\operatorname{supp} \mu$ can be thought of as a graph whose edges are smooth curve segments connecting certain vertices. The statement that $\operatorname{supp} \mu$ is connected and has connected complement then means that it is a connected graph without cycles, that is a tree. Recall that a connected graph is a tree precisely if the number of vertices exceeds the number of edges by exactly one.

Lemma 5. If the Cauchy transform of $\mu$ satisfies (11), then the support of $\mu$ is a tree. 
Proof. We will first prove that $\operatorname{supp} \mu$ is connected. To do this we will show that if $U$ is a bounded domain which is connected and simply connected, and the boundary of $U$ does not intersect $\operatorname{supp} \mu$, then either $\operatorname{supp} \mu \subset U$ or $\operatorname{supp} \mu \subset$ $\mathbf{C} \backslash U$. From this it easily follows that $\operatorname{supp} \mu$ is connected. Now it is clear that all the zeros of $Q_{k}$ are either contained in $U$ or in the complement of $U$, since $C(z)$ defines a continuous branch of $Q_{k}(z)^{-1 / k}$ along $\partial U$. Observe also that

$$
\frac{1}{2 \pi i} \int_{\partial U} C(z) d z=\frac{1}{2 \pi i} \int_{\mathbf{C}} \int_{\partial U} \frac{d z}{z-\zeta} d \mu(\zeta)=\int_{U} d \mu(\zeta)
$$

Now if all the zeros of $Q_{k}$ are contained in the complement of $U$, there is an analytic continuation of $C(z)$ across $U$, hence the left hand side of (12) vanishes. It follows that $\operatorname{supp} \mu \subset \mathbf{C} \backslash U$. If on the other hand, all the zeros of $Q_{k}$ are contained in $U$, then $C(z)$ has an analytic continuation in $\mathbf{C} \backslash U$ which is asymptotically equal to $a / z$ for some $k$ th root of unity $a$ when $z \rightarrow \infty$. Thus the left hand side of (12) is equal to $a$. Since the right hand side is positive, $a$ must be 1 , which means that all the mass of $\mu$ is in $U$. Hence we have proved that $\operatorname{supp} \mu$ is connected.

Now let $E$ be the set of all curve segments in $\operatorname{supp} \mu$ and let $V$ be the set of vertices which are endpoints of the edges in $E$. We may assume that $V$ contains all the zeros of $Q_{k}$. To every pair $e \in E, v \in V$ such that $v$ is an endpoint of $e$, we assign a number $\nu(e, v)$ by the following rule. Let $\gamma$ be a small loop winding once around $v$ in the clockwise direction, and let $\nu(e, v)$ be the jump of $(2 \pi i)^{-1} \log C(z)$ when $z$ crosses $e$ moving along $\gamma$. This number, which is defined modulo $\mathbf{Z}$, will be uniquely determined if we require that $0<\nu(e, v)<1$. Assume now that $v$ is not a zero of $Q_{k}$ and let $e_{1}, \ldots, e_{r}$ be the curves in $E$ having $v$ as one endpoint. (If some curve has both its endpoints in $v$, it will be counted twice.) Select a branch of $Q_{k}(z)^{1 / k}$ near $v$ and observe that by Lemma 3 and the proof of Lemma $4, Q_{k}(z)^{1 / k} C(z)$ is a $k$ th root of unity, which moves once around the unit circle in the counterclockwise direction as $z$ moves along $\gamma$. It follows that $\nu\left(e_{1}, v\right)+\cdots+\nu\left(e_{r}, v\right)=1$. If instead $v$ is a zero of $Q_{k}$ of multiplicity $m$, a slight modification of the argument shows that $\nu\left(e_{1}, v\right)+\cdots+\nu\left(e_{r}, v\right)=1-m / k$. On the other hand, it is clear that $\nu\left(e, v_{1}\right)+\nu\left(e, v_{2}\right)=1$ where $v_{1}, v_{2}$ are the endpoints of $e \in E$. Hence the sum of all the $\nu(e, v)$ is equal both to $\sharp V-1$ and to $\sharp E$. Since $\operatorname{supp} \mu$ is a connected graph, this implies that it is a tree.

We are now ready to prove the uniqueness part of Theorem 2. This is done by means of the following two lemmas.

Lemma 6. Suppose the Cauchy transform of $\mu$ satisfies (11) and let $u$ be the logarithmic potential of $\mu$. If $\Psi^{-1}$ is a (locally defined) inverse of a primitive function of $Q_{k}(z)^{-1 / k}$, then $u \circ \Psi^{-1}$ is convex. 
Proof. Let $\chi$ be as in the proof of Lemma 4. Since $2 \partial u / \partial z=C(z)$ we have

$$
\begin{aligned}
2 \frac{\partial}{\partial w} u\left(\Psi^{-1}(w)\right) & =2 \frac{\partial u}{\partial z}\left(\Psi^{-1}(w)\right) \cdot Q_{k}\left(\Psi^{-1}(w)\right)^{1 / k} \\
& =C\left(\Psi^{-1}(w)\right) \cdot Q_{k}\left(\Psi^{-1}(w)\right)^{1 / k} \\
& =\chi(w) .
\end{aligned}
$$

It follows from Corollary 1 , that $u \circ \Psi^{-1}$ is convex.

Lemma 7. Let $\mu$ be a measure whose Cauchy transform satisfies (11), let $\Omega=$ $\mathbf{C} \backslash \operatorname{supp} \mu$ and let $\Psi(z)$ be defined in $\Omega$ by

$$
\Psi(z)=\int \log (z-\zeta) d \mu(\zeta)
$$

Then $\Psi$ is a multivalued function mapping $\Omega$ onto a domain $H=\{w ; \operatorname{Re} w>$ $h(\operatorname{Im} w)\}$ where $h$ is a continuous function, and $\Psi^{-1}: H \rightarrow \Omega$ is a single valued function.

Proof. It is clear that $\Psi$ is a holomorphic function in $\Omega$ defined up to multiples of $2 \pi i$ and that $\Psi^{\prime}(z)=C(z)$. Let $\gamma$ be a curve segment of $\operatorname{supp} \mu$ and let $U$ be a one-sided neighbourhood of $\gamma$ in $\Omega$ on which $\Psi$ has a single valued branch. Now the restriction of $\Psi$ to $U$ has an analytic continuation across $\gamma$, and by Lemma $4, \Psi$ maps $\gamma$ to a line segment. Moreover, since in the notation of the proof of Lemma $4, \chi=1$ in $\Psi(U)$ and $\operatorname{Re} \chi \leq 1$ everywhere, it follows that $\Psi(\gamma)$ is not horizontal and that $\Psi(U)$ is on the right hand side of $\Psi(\gamma)$. Putting the segments $\Psi(\gamma)$ together as $U$ moves around $\operatorname{supp} \mu$, we obtain a broken line of the form $\{\operatorname{Re} w=h(\operatorname{Im} w)\}$ bounding a domain $H=\{\operatorname{Re} w>h(\operatorname{Im} w)\}$. It is clear that $\Psi$ maps $\Omega$ into $H$ and the boundary of $\Omega$ to the boundary of $H$. Now $\psi(z)=\exp (-\Psi(z))$ is a single valued proper mapping from $\Omega \cup\{\infty\}$ to $D=\{\zeta ; \log |\zeta|<-h(-\arg \zeta)\}$ which does not vanish in $\Omega$ and has a simple zero at $\infty$. It follows that $\psi: \Omega \cup\{\infty\} \rightarrow D$ is a bijection, hence $\Psi^{-1}(w)=\psi^{-1}\left(e^{-w}\right)$ is a single valued holomorphic mapping.

Corollary 3. If $\mu_{1}$ and $\mu_{2}$ are two probability measures whose Cauchy transforms satisfy (11), then $\mu_{1}=\mu_{2}$.

Proof. Let $\Psi$ be defined as in Lemma 7 with $\mu_{1}$ in place of $\mu$, and let $u_{1}$ and $u_{2}$ be the logarithmic potentials of $\mu_{1}$ and $\mu_{2}$. Then $u_{1}\left(\Psi^{-1}(w)\right)=\operatorname{Re} w$ for all $w \in H$ and $u_{2}\left(\Psi^{-1}(w)\right)=\operatorname{Re} w$ when $\operatorname{Re} w$ is sufficiently large. Since $u_{2} \circ \Psi^{-1}$ is convex by Lemma 6 , it follows that $u_{2}\left(\Psi^{-1}(w)\right) \geq \operatorname{Re} w$ for all $w \in H$, hence $u_{1}(z) \leq u_{2}(z)$ for almost all $z$. Similarly, $u_{2}(z) \leq u_{1}(z)$ for almost all $z$, and it follows that $\mu_{1}=\Delta u_{1} / 2 \pi=\Delta u_{2} / 2 \pi=\mu_{2}$.

\section{Root measures and the Cauchy transform}

In this section we describe the basic connections between root measures and the Cauchy transform which will be used to prove Theorem 4 . 
Let $\mu_{n}$ be a sequence of measures in the complex plane. The sequence is said to converge weakly to a measure $\mu$ if

$$
\int \phi(z) d \mu_{n}(z) \rightarrow \int \phi(z) d \mu(z)
$$

for every continuous function $\phi$ with compact support. If in addition there exists a compact set $K$ such that $\operatorname{supp} \mu_{n} \subset K$ for every $n$, we will say that $\mu_{n}$ converges weakly with compact support to $\mu$ and write $\mu_{n} \rightarrow \mu$ (w.c.s.).

If $K \subset \mathbf{C}$ is a compact set and $M(K)$ denotes the space of all probability measures with support in $K$, equipped with the weak topology, it is known that $M(K)$ is a sequentially compact Hausdorff space. We will use this fact to select a convergent subsequence from a sequence of measures as a first step in the proof of Theorem 4 .

If $\phi$ is a locally integrable function and $\mu$ is a compactly supported measure, the convolution

$$
(\phi * \mu)(z)=\int \phi(z-\zeta) d \mu(\zeta)
$$

is a locally integrable function defined almost everywhere in the complex plane. If $\mu_{n} \rightarrow \mu$ (w.c.s.), it is easy to show that $\phi * \mu_{n} \rightarrow \phi * \mu$ in $L_{l o c}^{1}$.

We will be particularly interested in the cases where $\phi(z)=\log |z|$ or $\phi(z)=1 / z$. Convolution with these functions defines the logarithmic potential

$$
u(z)=\int \log |z-\zeta| d \mu(\zeta)
$$

and the Cauchy transform

$$
C(z)=\int \frac{d \mu(\zeta)}{z-\zeta}
$$

of $\mu$. It is well known that the measure $\mu$ can be reconstructed from either $u$ or $C$ by the formula

$$
\mu=\frac{1}{2 \pi} \cdot \Delta u=\frac{1}{\pi} \cdot \frac{\partial C}{\partial \bar{z}}
$$

where $\Delta=(\partial / \partial x)^{2}+(\partial / \partial y)^{2}$ is the Laplace operator and $\partial / \partial \bar{z}=(\partial / \partial x+$ $i \partial / \partial y) / 2$. These identities should be understood in the sense of distribution theory.

Let $p$ be a polynomial of degree $n$ and let $\mu$ be the root measure of $p$, as defined in the introduction. If $p$ is monic, the logarithmic potential of $\mu$ is given by

$$
\frac{1}{n} \log |p(z)|=\int \log |z-\zeta| d \mu(\zeta),
$$

and for any $p$, the Cauchy transform of $\mu$ is

$$
\frac{p^{\prime}(z)}{n p(z)}=\int \frac{d \mu(\zeta)}{z-\zeta}
$$


These two identities, which can easily be verified, are among the main ingredients in the proof of Theorem 4 . We will here use them to prove a general lemma which will be needed later.

Lemma 8. Let $p_{m}$ be a sequence of polynomials, such that $n_{m}:=\operatorname{deg} p_{m} \rightarrow \infty$ and let $\mu_{m}$ and $\mu_{m}^{\prime}$ be the root measures of $p_{m}$ and $p_{m}^{\prime}$ respectively. If $\mu_{m} \rightarrow \mu$, $\mu_{m}^{\prime} \rightarrow \mu^{\prime}$ (w.c.s.) and $u$ and $u^{\prime}$ are the logarithmic potentials of $\mu$ and $\mu^{\prime}$, then $u^{\prime} \leq u$ with equality in the unbounded component of $\mathbf{C} \backslash \operatorname{supp} \mu$.

Proof. Assume with no loss of generality that $p_{m}$ are monic. Let $K$ be a compact set containing the zeros of every $p_{m}$. By $(13)$ we then have

$$
u(z)=\lim _{m \rightarrow \infty} \frac{1}{n_{m}} \log \left|p_{m}(z)\right|
$$

and

$$
u^{\prime}(z)=\lim _{m \rightarrow \infty} \frac{1}{n_{m}-1} \log \left|\frac{p_{m}^{\prime}(z)}{n_{m}}\right|=\lim _{m \rightarrow \infty} \frac{1}{n_{m}} \log \left|\frac{p_{m}^{\prime}(z)}{n_{m}}\right|
$$

with convergence in $L_{l o c}^{1}$. Hence by (14),

$$
u^{\prime}(z)-u(z)=\lim _{m \rightarrow \infty} \frac{1}{n_{m}} \log \left|\frac{p_{m}^{\prime}(z)}{n_{m} p_{m}(z)}\right|=\lim _{m \rightarrow \infty} \frac{1}{n_{m}} \log \left|\int \frac{d \mu_{m}(\zeta)}{z-\zeta}\right| .
$$

Now, if $\phi$ is a positive test function it follows that

$$
\begin{aligned}
\int \phi(z)\left(u^{\prime}(z)-u(z)\right) d \lambda(z) & =\lim _{m \rightarrow \infty} \frac{1}{n_{m}} \int \phi(z) \log \left|\int \frac{d \mu_{m}(\zeta)}{z-\zeta}\right| d \lambda(z) \\
& \leq \lim _{m \rightarrow \infty} \frac{1}{n_{m}} \int \phi(z) \int \frac{d \mu_{m}(\zeta)}{|z-\zeta|} d \lambda(z) \\
& =\lim _{m \rightarrow \infty} \frac{1}{n_{m}} \iint \frac{\phi(z) d \lambda(z)}{|z-\zeta|} d \mu_{m}(\zeta)
\end{aligned}
$$

where $\lambda$ denotes Lebesgue measure in the complex plane. Since $1 /|z|$ is locally integrable, the function $\int \phi(z)|z-\zeta|^{-1} d \lambda(z)$ is continuous, and hence bounded by a constant $M$ for all $z$ in $K$. Since supp $\mu_{m} \subset K$, the last expression in (16) is bounded by $M / n_{m}$, hence the limit when $m \rightarrow \infty$ is 0 . This proves that $u^{\prime} \leq u$.

In the complement of $\operatorname{supp} \mu, u$ is harmonic and $u^{\prime}$ is subharmonic, hence $u^{\prime}-u$ is a negative subharmonic function. Moreover, in the complement of $K, p_{m}^{\prime} /\left(n_{m} p_{m}\right)$ converges uniformly on compact sets to the Cauchy transform $C(z)$ of $\mu$. Since $C(z)$ is a nonconstant holomorphic function in the unbounded component of $\mathbf{C} \backslash K$, it follows from (15) that $u^{\prime}-u=0$ there. By the maximum principle for subharmonic functions it follows then that $u^{\prime}-u=0$ in the unbounded component of $\mathbf{C} \backslash \operatorname{supp} \mu$. The proof is complete.

\section{Root measures of eigenpolynomials}

We now turn to the proof of Theorem 4 . The plan is to show that $\mu_{n}$ converges to a measure whose Cauchy transform satisfies (11). This will prove Theorem 4 and the existence part of Theorem 2. Let $\mu_{n}$ be the root measure of $p_{n}$ as in the 
statement of Theorem 4. Also let $\mu_{n}^{(i)}$ be the root measure of the $i$ th derivative $p_{n}^{(i)}$. We begin by showing that there is a compact set $K$ containing the supports of all the measures $\mu_{n}^{(i)}$.

Lemma 9. Let $Q_{0}, \ldots, Q_{k}$ be fixed and let $p_{n}$ be an eigenpolynomial of degree $n$ of the operator $T_{Q}$. Then there exists a compact set $K$ such that all the zeros of every $p_{n}^{(i)}$ lie in $K$ for every $n$ and every $i \geq 0$. If $Q_{0}=\ldots=Q_{k-1}=0, K$ may be taken as the convex hull of the zeros of $Q_{k}$.

Proof. The case with $Q_{0}=\cdots=Q_{k-1}=0$ was treated in [4]. In the general case it suffices to check the roots of $p_{n}$, since by Gauss-Lucas' theorem the roots of any derivative $p_{n}^{(i)}$ are contained in the convex hull of the roots of $p_{n}$. Furthermore it suffices to show that there exists a compact set containing the zeros of $p_{n}$ for large values of $n$, since for any finite value of $n$ we have finitely many roots of the polynomial $p_{n}$, and these are clearly contained in some compact set.

Let $z$ be a root of $p_{n}$. Then

$$
T_{Q}\left(p_{n}\right)(z)=\sum_{i=0}^{k} Q_{i}(z) \cdot p_{n}^{(i)}(z)=\lambda_{n} \cdot p_{n}(z)=0
$$

or, equivalently,

$$
Q_{k}(z) \cdot p_{n}^{(k)}(z)+Q_{k-1}(z) \cdot p_{n}^{(k-1)}(z)+\ldots+Q_{1}(z) \cdot p_{n}^{(1)}(z)=0 .
$$

We will show that for sufficiently large choices of $|z|$ and $n$ this equation will not hold. It is possible to find some $r_{0}$ and some $n_{0}$ such that if $|z| \geq r_{0}$ and $n>n_{0}$ then $z$ cannot be a root of $p_{n}$. Using formula (14) we have

$$
\frac{p_{n}^{(i+1)}(z)}{(n-i) \cdot p_{n}^{(i)}(z)}=\int \frac{d \mu_{n}^{(i)}(\zeta)}{z-\zeta}=: b_{i} .
$$

Thus

$$
\begin{gathered}
p_{n}^{(k-1)}(z)=\frac{p_{n}^{(k)}(z)}{(n-k+1) \cdot b_{k-1}}, \\
p_{n}^{(k-2)}(z)=\frac{p_{n}^{(k-1)}(z)}{(n-k+2) \cdot b_{k-2}}=\frac{p_{n}^{(k)}(z)}{(n-k+1)(n-k+2) \cdot b_{k-1} \cdot b_{k-2}},
\end{gathered}
$$

and so on. Generally we have

$$
p_{n}^{(i)}(z)=\frac{p_{n}^{(k)}(z)}{(n-k+1) \ldots(n-i) \cdot \prod_{j=i}^{k-1} b_{j}} .
$$

Now assume that $z$ is the root of $p_{n}$ with the largest modulus and let $|z|=r$. With $\zeta$ being a root of some $p_{n}^{(i)}$ we have $|\zeta| \leq|z|$ by Gauss-Lucas' theorem. We 
will estimate $b_{i}=\int \frac{d \mu_{n}^{(i)}(\zeta)}{z-\zeta}$ so that $\left|b_{i}\right| \geq 1 / 2 r \quad \forall i \leq k$. We have

$$
\frac{1}{z-\zeta}=\frac{1}{z} \cdot \frac{1}{1-\zeta / z}=\frac{1}{z} \cdot \frac{1}{1-\theta}
$$

and $|\theta|=|\zeta / z| \leq 1$.

With $w=1 /(1-\theta)$ we obtain

$$
\begin{gathered}
|w-1|=\left|\frac{1}{1-\theta}-\frac{(1-\theta)}{(1-\theta)}\right|=\frac{|\theta|}{|1-\theta|}=|\theta||w| \leq|w| \\
\Leftrightarrow \\
|w-1| \leq|w| \\
\Leftrightarrow \\
\operatorname{Re}(w) \geq \frac{1}{2} .
\end{gathered}
$$

Using this result we get

$$
\begin{aligned}
\left|b_{i}\right| & =\left|\int \frac{d \mu_{n}^{(i)}(\zeta)}{z-\zeta}\right|=\frac{1}{r}\left|\int \frac{d \mu_{n}^{(i)}(\zeta)}{1-\theta}\right|= \\
& =\frac{1}{r}\left|\int w d \mu_{n}^{(i)}(\zeta)\right| \geq \frac{1}{r}\left|\int \operatorname{Re}(w) d \mu_{n}^{(i)}(\zeta)\right| \geq \\
& \geq \frac{1}{2 r} \int d \mu_{n}^{(i)}(\zeta)=\frac{1}{2 r} .
\end{aligned}
$$

Now we choose $r_{0}$ in such a way that $\left|Q_{k}(w)\right| \geq r^{k} / 2$ as $|w| \geq r_{0}$ and then a constant $C$ such that $\left|Q_{i}(w)\right| \leq C \cdot r^{i}$ for every $i=1, \ldots, k-1$. Finally we choose $n_{0}$ such that $\frac{C \cdot 2^{k-i+1}}{(n-i) \ldots(n-k+1)}<\frac{1}{k-1}$ as $n>n_{0}$ for every $i=1, \ldots, k-1$. Then, as $|z|=r \geq r_{0}$ and $n>n_{0}$, we get

$$
\begin{aligned}
\left|\frac{Q_{i}(z) \cdot p_{n}^{(i)}(z)}{Q_{k}(z) \cdot p_{n}^{(k)}(z)}\right| & =\frac{\left|Q_{i}(z)\right|}{\left|Q_{k}(z)\right|} \cdot \frac{(n-k) !}{(n-i) !} \cdot \frac{1}{\prod_{j=i}^{k-1}\left|b_{j}\right|} \leq \\
& \leq \frac{\left|Q_{i}(z)\right|}{\left|Q_{k}(z)\right|} \cdot \frac{(n-k) !}{(n-i) !} \cdot 2^{k-i} \cdot r^{k-i} \leq \\
& \leq \frac{C \cdot r^{i}}{r^{k} / 2} \cdot \frac{(n-k) !}{(n-i) !} \cdot 2^{k-i} \cdot r^{k-i}= \\
& =\frac{C \cdot 2^{k-i+1}}{(n-i) \ldots(n-k+1)}<\frac{1}{k-1} .
\end{aligned}
$$

Dividing (17) by $Q_{k}(z) \cdot p_{n}^{(k)}(z)$ we obtain

$$
1+\sum_{i=1}^{k-1} \frac{Q_{i}(z) \cdot p_{n}^{(i)}(z)}{Q_{k}(z) \cdot p_{n}^{(k)}(z)}=0
$$


but with $r \geq r_{0}$ and $n>n_{0}$ we get

$$
\left|\sum_{i=1}^{k-1} \frac{Q_{i}(z) \cdot p_{n}^{(i)}(z)}{Q_{k}(z) \cdot p_{n}^{(k)}(z)}\right| \leq \sum_{i=1}^{k-1}\left|\frac{Q_{i}(z) \cdot p_{n}^{(i)}(z)}{Q_{k}(z) \cdot p_{n}^{(k)}(z)}\right|<\sum_{i=1}^{k-1} \frac{1}{k-1}=1
$$

and so (17) cannot be fulfilled with such choices of $r$ and $n$.

Assume that $N$ is a subsequence of the natural numbers such that

$$
\mu^{(j)}=\lim _{n \rightarrow \infty, n \in N} \mu_{n}^{(j)}
$$

exists for $j=0, \ldots, k$. The following lemma shows that the Cauchy transform of $\mu=\mu^{(0)}$ satisfies (11).

Lemma 10. The measures $\mu^{(j)}$ are all equal and the Cauchy transform $C(z)$ of this common limit satisfies $C(z)^{k}=1 / Q_{k}(z)$ for almost every $z$.

Proof. By (14) we have that

$$
\frac{p_{n}^{(j+1)}(z)}{(n-j) p_{n}^{(j)}(z)} \rightarrow \int \frac{d \mu^{(j)}(\zeta)}{z-\zeta}
$$

with convergence in $L_{l o c}^{1}$, and by passing to a subsequence once again we can assume that we have pointwise convergence almost everywhere. From the relation $T_{Q} p_{n}=\lambda_{n} p_{n}$ it follows that

$$
\begin{aligned}
Q_{k} \frac{p_{n}^{(k)}}{n \ldots(n-k+1) p_{n}}= & \frac{\lambda_{n}}{n \ldots(n-k+1)} \\
& -\sum_{l=0}^{k-1} \frac{Q_{l}}{(n-l) \ldots(n-k+1)} \prod_{j=0}^{l-1} \frac{p_{n}^{(j+1)}}{(n-j) p_{n}^{(j)}} .
\end{aligned}
$$

Now $\lambda_{n} / n \ldots(n-k+1) \rightarrow 1$ by Theorem 1 , while the sum converges pointwise to 0 almost everywhere by virtue of the factors $(n-l) \ldots(n-k+1)$ in the denominators. It follows that

$$
\frac{p_{n}^{(k)}(z)}{n \ldots(n-k+1) p_{n}(z)} \rightarrow \frac{1}{Q_{k}(z)}
$$

when $n \rightarrow \infty$ through the sequence $N$ for almost every $z$. If $u^{(j)}$ denotes the logarithmic potential of $\mu^{(j)}$, then it follows from (13) and (21) that

$$
u^{(k)}-u^{(0)}=\lim _{n \rightarrow \infty} \frac{1}{n} \log \left|\frac{p_{n}^{(k)}}{n \ldots(n-k+1) p_{n}}\right|=-\lim _{n \rightarrow \infty} \frac{1}{n} \log \left|Q_{k}\right|=0 .
$$

On the other hand we have from Lemma 8 that $u^{(0)} \geq u^{(1)} \geq \cdots \geq u^{(k)}$, hence the potentials $u^{(j)}$ are all equal, and it follows that $\mu^{(j)}=\Delta u^{(j)} / 2 \pi$ are all equal. Finally we have from (19) and (21) that

$$
C(z)^{k}=\lim _{n \rightarrow \infty} \prod_{j=0}^{k-1} \frac{p_{n}^{(j+1)}(z)}{(n-j) p_{n}^{(j)}(z)}=\lim _{n \rightarrow \infty} \frac{p_{n}^{(k)}(z)}{n \ldots(n-k+1) p_{n}(z)}=\frac{1}{Q_{k}(z)}
$$


for almost every $z$. This completes the proof.

Corollary 4. There exists a unique measure $\mu_{Q_{k}}$ satisfying the requirements in Theorem 2. The sequence $\mu_{n}$ converges weakly to $\mu_{Q_{k}}$. Moreover, supp $\mu_{Q_{k}}$ is contained in the convex hull of the zeros of $Q_{k}$.

Proof. By Theorem 1, the operator $T_{Q}$ has an eigenpolynomial $p_{n}$ of degree $n$ for all sufficiently large $n$. By Lemma 9 , there exists a compact set $K$ such that $\operatorname{supp} \mu_{n}^{(j)} \subset K$ for all $n$. By compactness, there exists a subsequence $N$ such that the limit (18) exists for $j=0, \ldots, k$. By Lemma $10, \mu_{Q_{k}}=\mu^{(0)}$ has the required properties, so existence is proved. Uniqueness was established in section 3 . Since we may take $Q_{0}=\ldots=Q_{k-1}=0$, and in this case $\operatorname{supp} \mu_{n}^{(j)} \subset K$ where $K$ is the convex hull of the zeros of $Q_{k}$ by Lemma 9 , it follows that $\operatorname{supp} \mu_{Q_{k}}$ is also contained in $K$.

Assume that $\mu_{n}$ does not converge to $\mu_{Q_{k}}$ Then we can find a subsequence $N^{\prime}$ of the natural numbers such that $\mu_{n}$ stays away from a fixed neighbourhood of $\mu_{Q_{k}}$ in the weak topology, for all $n \in N^{\prime}$. Again by compactness, we can find a subsequence $N$ of $N^{\prime}$ such that the limit (18) exists for $j=0, \ldots, k$. By Lemma 10 and the uniqueness of $\mu_{Q_{k}}$, it follows that $\mu^{(0)}=\mu_{Q_{k}}$, contradicting the assumption that $\mu_{n}$ stays away from $\mu_{Q_{k}}$ for all $n$ in $N^{\prime}$ and hence all $n$ in $N$. The proof is complete.

\section{References}

[1] L. Hörmander, The analysis of linear partial differential operators. I, Distribution theory and Fourier analysis. Second edition. Springer Study Edition. Springer-Verlag, Berlin, 1990.

[2] E. Kamke, Differentialgleichungen. Lösungsmetoden und Lösungen, Becker \& Erler, Leipzig, 1942.

[3] K. Kwon, L. Littlejohn, G. Yoon, Bochner-Krall orthogonal polynomials, Special functions (Hong Kong, 1999), 181-193, World Sci. Publishing, River Edge, N. J., 2000.

[4] G. Masson, B. Shapiro, On polynomial eigenfunctions of a hypergeometric-type operator, to appear in Experimental Mathematics.

[5] Thomas Ransford, Potential theory in the complex plane, London Mathematical Society Student Texts, 28. Cambridge University Press, Cambridge, 1995.

[6] Harold Shapiro, Spectral aspects of a class of differential operators, to appear in Proceedings of S. Kovalevsky conference held in Stockholm, June 2000, to be published in Operator Theory: Advances and Applications, Birkhäuser, 2002.

Matematiska institutionen, Stockholms universitet, SE-10691 Stockholm, SWEDEN.

E-mail address: tanjab@matematik.su.se

E-mail address: hansr@matematik.su.se 